\title{
Thrombolysis versus thrombectomy in acute deep vein thrombosis
}

Deep vein thrombosis (DVT) is a significant health problem leading to the admission of over 250,000 Americans each year. Its most serious acute complication, namely pulmonary embo is kills approximately 100,000 each year, and is the third most common cardiovascular related mortanty after myocardial infarction and stroke. Chronic leg problems following DVT include leg heaviness, tiredness, cramping and ulceration. These are termed the post-thrombotic syndrome. The current standard therapy of anticoagulation has changed little over 50 years. It does not remove or destroy thrombus, relying instead on the bodies own fibrinolytic mechanisms to do so. DVT needs to be more accurately categorized on an anatomical basis, and for a variety of reasons, the area of most importance is the iliofemoral region. The rationale for active, rather than passive, thrombus removal relies on multiple observations that, by doing so improves luminal patency, restores valvular function and has the potential to reduce the severity of post-thrombotic syndrome. Techniques of thrombus removal include catheter-directed thrombolysis, mechanical thrombectomy, and various combinations of both (pharmacomechanical catheter-directed thrombolysis). Although there no few direct trials comparing these directly, there is a reasonable body of evidence demonstrating their efficacy.

KEYWORDS: catheter directed thrombolysis deep vein thrombosis intervention pharmacomechanical venous thrombectomy post-thrombotic syndrome ultrasound

Acute deep vein thrombosis (DVT) is a major health issue causing approximately 300,000600,000 new cases per annum in the USA [101] Venous thromboembolism accounts for more deaths than the total combined mortality of breast cancer, road traffic accidents and AIDS combined [101]. It is the third most common cause of cardiovascular mortality after myocardial infarction and cerebral vascular accidents. Apart from the initial symptoms, which may be severe and progress to phlegmasia cerulea dolens, there is the risk of pulmonary embolic disease that can be life threatening. The chronic subsequent leg problems of leg swelling, pain, ulceration and so on are termed the 'post-thrombotic syndrome'.

Conventional management of acute DVT is based upon one trial from 1960 and several subsequent observational trials [1-3]. It has changed little over that time. This consists of immediate anticoagulation with heparin or, more recently, low-molecular-weight heparin followed by 3 months of oral anticoagulation. However, neither of these treatments offers significant fibrinolytic activity, relying instead on the body's own Urokinase.

Acute DVT refers to symptoms less than 14 days.

\section{The case for more accurate anatomical categorization of DVT}

Iliofemoral DVT (IF DVT) refers to occlusive or partial thrombosis between the IVC and common femoral vein (CFV). This segment is critical since if the femoral vein of the thigh occludes, blood travels from the calf up to the popliteal and then, via profunda collaterals, up to the CFV and so on up to the IVC. Hence a proportion of patients who develop acute DVT of the mid-femoral vein may not suffer postthrombotic morbidity. As with arterial disease, if the profunda femoris is patent, an occluded femoral vein between the adductor canal and the inflow of the profunda femoris into the CFV may cause minimal morbidity. On the other hand, an obliterated common femoral or iliac venous system results in poor drainage of the whole lower extremity venous system as it is the single venous outflow channel for the entire leg [4]. Blood has to drain via inefficient cross pelvic or deep pelvic collaterals, leading to raised venous pressures and eventually severe post-thrombotic morbidity.

One of the problems with the existing literature is that patients are not well stratified into different segments of the venous anatomy, thus those with a femoral popliteal DVT are placed
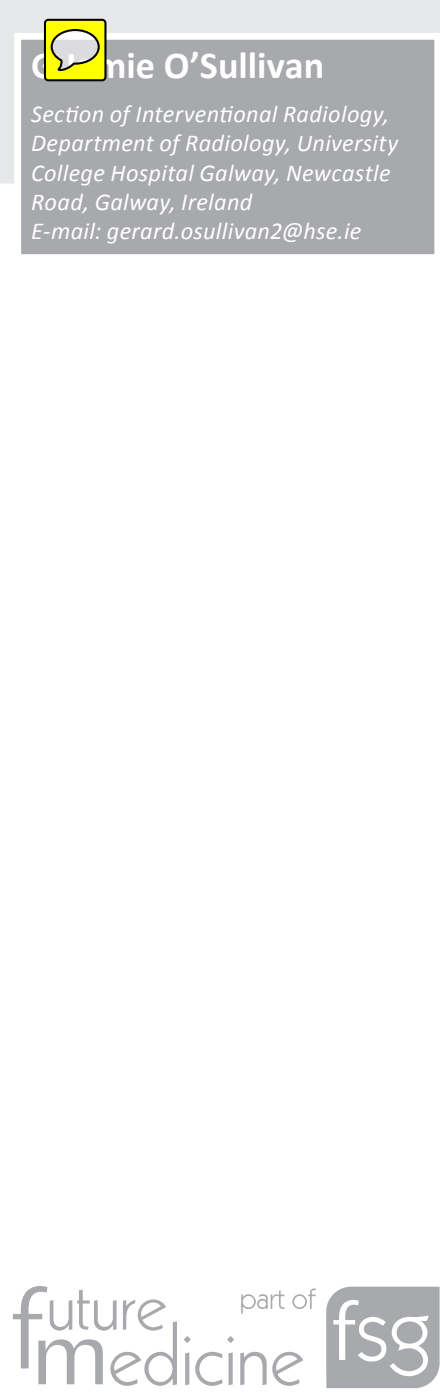


\section{Table 1. Medical-surgical interventions to treat deep vein thrombosis.}

\begin{tabular}{|c|c|c|c|c|}
\hline Approaches & $\begin{array}{l}\text { Indications for } \\
\text { DVT }\end{array}$ & Technical purpose & Considerations & Ref. \\
\hline Systemic anticoagulation & $\begin{array}{l}\text { Accepted } \\
\text { conservative gold } \\
\text { standard for all } \\
\text { DVT }\end{array}$ & $\begin{array}{l}\text { Prevents further propagation } \\
\text { of thrombus or PE }\end{array}$ & $\begin{array}{l}\text { Does not remove thrombus; ? does not inhibit } \\
\text { development of PTS; bleeding rate } 2-4 \% \\
\text { When treated with anticoagulant alone, patients } \\
\text { with iliofemoral DVT continue to have high } \\
\text { incidence of chronic painful edema ( } 75 \% \text { ) and } \\
\text { venous claudication ( } 40 \%)\end{array}$ & {$[4]$} \\
\hline Systemic thrombolysis & $\begin{array}{l}\text { No indication for } \\
\text { isolated DVT } \\
\text { Useful in PE }\end{array}$ & $\begin{array}{l}\text { Accelerates thrombus lysis } \\
\text { indiscriminately throughout } \\
\text { body }\end{array}$ & $\begin{array}{l}\text { Major bleeding rate is risky and extremely } \\
\text { variable. High dependency monitoring needed } \\
\text { over hours or days }\end{array}$ & {$[8]$} \\
\hline $\begin{array}{l}\text { Surgical thrombectomy } \\
\text { Open venous } \\
\text { Balloon }\end{array}$ & $\begin{array}{l}\text { Cases refractory to } \\
\text { less invasive } \\
\text { interventions }\end{array}$ & Removes thrombus ${ }^{\dagger}$ & $\begin{array}{l}\text { Invasive; requires general anesthesia; may delay } \\
\text { stenting; slower mobilization and longer recovery } \\
\text { post-surgery; experience limited to a few centers }\end{array}$ & {$[19]$} \\
\hline $\begin{array}{l}\text { CDT } \\
\text { Simple } \\
\text { Ultrasound-assisted } \\
\text { idowave }{ }^{\circledR} \text { ) }\end{array}$ & $\begin{array}{l}\text { Serious DVT and/or } \\
\text { PE }\end{array}$ & $\begin{array}{l}\text { Directs iv. thrombolytic agent } \\
\text { Q e site of the thrombus }\end{array}$ & $\begin{array}{l}\text { ICU monitoring } 24-72 \text { (average } 48 \text { ) h; lytic agent } \\
\text { migrates systemically; potential hemorrhage; } \\
\text { major bleeding rate } 5-11 \% \\
\text { US-aided: CDT time is reduced to median } 22 \mathrm{~h} \\
\text { and bleeding rate to } 3.8 \%\end{array}$ & {$[43]$} \\
\hline $\begin{array}{l}\text { Percutaneous mechanical } \\
\text { thrombectomy (Trerotola } \\
\text { and Angiojet }{ }^{\circledR} \text { ) }\end{array}$ & $\begin{array}{l}\text { When } \\
\text { thrombolytics are } \\
\text { absolutely } \\
\text { contraindicated }\end{array}$ & $\begin{array}{l}\text { Removes thrombus with action } \\
\text { of rotary, scraping, or } \\
\text { high-powered water jets }\end{array}$ & Potential vein wall damage and hemolysis & {$[46]$} \\
\hline $\begin{array}{l}\text { Pharmacomechanical } \\
\text { thrombolysis (Angiojet }{ }^{\circledR} \text { ) }\end{array}$ & $\begin{array}{l}\text { Single-session } \\
\text { treatment of DVT }\end{array}$ & $\begin{array}{l}\text { High-powered water jets } \\
\text { increase action of iv. } \\
\text { thrombolytic agent directed to } \\
\text { the site of the thrombus }\end{array}$ & $\begin{array}{l}\text { Potential hemolysis; proximal to hed } \\
\text { associated with microemboli and braaycardia } \\
\text { Systemic release of lytic agent } \\
\text { Day fail to remove much of the thrombus }\end{array}$ & {$[47]$} \\
\hline $\begin{array}{l}\text { Isolated pharmaco- } \\
\text { mechanical thrombolysis } \\
\text { (Trellis) }\end{array}$ & $\begin{array}{l}\text { Single-session } \\
\text { treatment of DVT }\end{array}$ & $\begin{array}{l}\text { Macerating wire increases } \\
\text { action of iv. thrombolytic } \\
\text { agent directed to the site of } \\
\text { the thrombus }\end{array}$ & $\begin{array}{l}\text { Isolates lytic agent, restricting systemic release } \\
\text { Requires an } 8 \mathrm{~F} \text { sheath so cannot really be used } \\
\text { below mid calf; has a small-caliber aspiration } \\
\text { lumen }\end{array}$ & {$[48]$} \\
\hline
\end{tabular}

in essentially the same category as those with an obstructed iliofemoral venous segment [5-8] .

Spontaneous recanalization of iliofemoral deep vein segments is very poor with anticoagulation alone [5].

The outcome for the two groups of patients is considerably different; those patients with iliofemoral DVT essentially represent a separate set of patients with markedly increased post-thrombotic morbidity and, therefore, warrant special treatment-related considerations [9].

The landmark paper by O'Donnell in 1977 demonstrated the severe long-term symptoms that iliofemoral DVT patients suffered with a high incidence of post-thrombotic syndrome, ulceration and inability to work [10]. Undoubtedly, the clinical outcome has improved somewhat with compression hosiery (if worn properly) but nonetheless adherence is poor particularly in warm climates.

Given that iliofemoral DVT gives the highest incidence of PTS, attention over the years has been primarily focused on treating this set of patients [11,12].
This review is directed squarely at this subset of venous thrombosis.

\section{Rationale for active thrombus removal}

The rationale to remove thrombus from the deep veins of patients with acute DVT is to restore patency, preserve valvular function (the valves are otherwise destroyed by venous thrombosis, scarring and wall thickening) and thereby avoid post-thrombotic morbidity [4,13,14]. It has been repetitively shown in multiple trials that thrombolysis and/or thrombectomy improves the rate of patency of the iliofemoral venous segment $[4,15]$.

In addition, there are considerable data that early thrombus removal results in diminished PISIF DVT. It has also been shown that the greater the proportion of thrombus removal, the better the venous patency and reduction in PTS subsequently.

A Cochrane review [16] comparing catheterdirected thrombolysis (CDT) for acute DVT with traditional anticoagulation in nearly 
700 patients revealed the following:

- Significantly improved early clot lysis with a relative risk reduction of 4.14 ; $95 \% \mathrm{CI}: 1.22-$ 14.01 to late clot lysis (RR: 2.71 ; $95 \%$ CI: $1.84-$ 3.99).

- Reduced post-thrombotic syndrome (RR: 0.66; 95\% CI: 0.47-0.94).

This latter issue is critical to the entire treatment algorithm, as there is as yet, no level-one evidence th 10 occurs with proximal DVT. This is the purpose of the ATTRACT study [101], a multicenter NIH-sponsored trial comparing a strategy of thrombus removal (interventional treatments, thrombolysis/thrombectomy) versus anticoagulation alone in patients with symptomatic proximal DVT, stratifying patients with iliofemoral or femoral popliteal DVT. The primary end point of this trial is to assess whether PCI D educes post-thrombotic syndrome; improves quality of life, is cost effective and is safe. Secondary end points include valvular patency, recurrent VTE and death. Currently recruiting, (aim is for 692 patients) results may be available by 2016 .

To summarize, patients with iliofemoral DVT suffer the greatest post-thrombotic sequelae; aggressive, early and successful treatment of this segment by thrombus removal/dissoluti $\cap$ elds the greatest clinical benefit.

\section{Current treatment recommendations}

Although not the focus of this article, readers are encouraged to review the recommendations published relatively recently in Chest and Circulation concerning the optimal anticoagulation regimens for acute IF DVT [8,9].

In essence, patients with IFDVT who receive warfarin must have it overlapped with initial AC (usually heparin or low-molecular-weight heparin) until INR $>2$ for at least $24 \mathrm{~h}$ with a target INR of $2-3$. If the IF DVT was related to a major reversible risk factor, then $3 / 12$ treatment is sufficient, if not, they require $6 / 12$ or life. A hematology workup may be sensible. Oncology patients should receive LMWH for as long as their cancer is active.

In terms of thrombolysis, Chest recommended that in acute IF DVT, in selected patients with symptoms of less than 14 days, reasonable life expectancy, good functional status, CDT may be used to reduce acute symptoms and postthrombotic morbidity if appropriate expertise and resources are available (Grade 2B).

After successful CDT in patients with acute DVT, the same intensity and duration
Box 1. Accelerated thrombus removal mechanisms (all with aspiration of slurry).

- Rotational mechanical. A fragmentation cage pulled through the vein macerates and strips thrombus from the vein walls (Trerotola Arrow; PA, USA) [26]

- Isolated pharmacomechanical thrombolysis. Balloons largely confine the treatment area, where a thrombolytic agent is dispersed through thrombus with a rotating wire (Trellis, Covidien; CA, USA) [51]

- Bernoulli's principle effect. High-powered jets fragment the thrombus into microscopic pieces (Angiojet ${ }^{\circledR}$, Medrad Interventional/Possis; PA, USA) [29]

- US-assisted catheter-directed thrombolysis. US waves partially fragment thrombus that can be attacked by a thrombolytic agent (EndoWave ${ }^{\text {TM/EkoSoni }}{ }^{\circledR}$, Ekos $^{\circledR}$, WA, USA) [36]

of anticoagulant therapy as for comparable patients who do not undergo CDT (Grade 1C) is recommended.

\section{CDT}

Most interventionalists are comfortable with CDT in the peripheral or coronary arteries for acute thrombosis. CDT has been used successfully for nearly 30 years in this context, Results of an American multicenter regist \& published in 1998 [17] and although somewhat disparate (multiple centers, different treatment algorithms) they were helpful in terms of identification of potential risks and complications. Essentially, the risk of significant intracranial bleed is considerably less than $1 \%$; the risk of a GI bleed requiring transfusion i a he order of $5 \%$.

\section{Technique CDT}

The technique is relatively straightforward. Access is gained to the obstructed venous segment or preferably below it, the catheter is threaded through the thrombus and infusion of thrombolysis is commenced. Alteplase is the thrombolytic most commonly employed but there is no evidence that this is superior to any other thrombolytic. The dosage is of the order of $1 \mathrm{mg} / \mathrm{h}$, while the patient is on strict bed rest and is also fully anticoagulated. After a variable period (typically of the order of 30 to $60 \mathrm{~h}$ ) and multiple interval venograms to assess progress, completion venography is performed, and usually an underlying stenotic lesion revealed which will require balloon angioplasty and stenting.

Although successful and probably the preferred technique currently employed by most institutions, there are a number of problems associated with this technique. Most institutions require that the patient be cared for in a high dependency unit where monitoring of neurological function and hemodynamic stability occurs whilst on thrombolysis. This results in 
a markedly increased cost as the typical stay in this unit is 2-3 days. In addition, although not a linear effect, the incidence of complications for CDT is related to both the time and total dose 9 PA (or whatever other thrombolytic agent used).

\section{Percutaneous mechanical thrombectomy devices}

In essence, all of these devices are used to decrease the time taken for thrombus removal and possibly to reduce the risk of prolonged infusion.

Percutaneous mechanical thrombectomy (PMT) devices may be categorized as rotational, rheolytic or ultrasound enhanced.

Rotational devices such as the Trerotola device (Arrow International, PA, USA) and the Amplatz thrombectomy device (Microvena, MN, USA) employ a high velocity rotating helix to macerate up the thrombus.

The Trellis device (Covidien, Bacchus Vascular, CA, USA) employs an oscillating rather than rotating sinusoidal nitinol wire between proximal and distal balloons while at the same time infusing thrombolytic agents in the segment 'isolated' by the balloon.

The AngioJet ${ }^{\circledR}$ device (Possis, MN, USA) generates a high-pressure saline jet to create a pressure gradient resulting in rheolytic thrombectomy with aspiration of the softened thrombus into the catheter.

Ultrasound assisted devices, EKOS ${ }^{\circledR}$ Endowave $^{\mathrm{TM}}$ device (EKOS Corporation, WA, USA), contains multiple ultrasound transducers that emit high frequency, low energy ultrasound energy in a radial fashion to enhance the penetration of thrombolysis by exposing plasminogen receptor sites. This technique probably has less of an hemolytic effect than saline pressure thrombectomy and possible less endothelial damage than rotational thrombectomy devices. It does suffer the disadvantage that it is not a single session technique, employing typical 16-25 h for treatment of a possible iliofemoral DVT (considerably shorter than the 40-60 h for conventional CDT but obviously slower than mechanical thrombectomy).

\section{Procedure of PMT}

The Society of Interventional Radiology has graded procedural success in relation to the luminal patency $[18,19]$ :

- Grade 1 SIR Thrombolysis-less than 50\% thrombus removal.
- Grade 2 SIR Thrombolysis-50 to 95\% thrombus removal.

- Grade 3 SIR Thrombolysis-greater than 95\%.

These are quite broad ranges but is difficult to be more precise because of the degree of subjectivity in estimating thrombus removal based on pre- and post-thrombolysis/thrombectomy venograms. 50\% luminal patency post-thrombolysis has been shown to correlate with significantly improved 1 year patency [11]. In practical terms, a combination of luminal patency and rapidity of inline flow as well as the abolition of collaterals appears to demonstrate the best success. With practice and experience, SIR Grade 2/3 can be achieved in the majority of acute DVT in a single session if the popliteal vein is clear.

A total of 16 retrospective case studies have described the use of PMT with or without CDT in a little under 500 patients [20-35,102], two of these studies were retrospective comparative studies comparing PMT plus CDT versus CDT alone. To date, there have been no randomized clinical trials of PMT compared with standard anticoagulation. Four ongoing studies have not published their results to date; the CAVA trial, the Sonic 1 Safety and Efficacy trial, the PEARL registry, but, most importantly, the ATTRACT study [36,103-105].

PMT has been reported as being successful on its own without CDT. It is recognized that the administration of CDT alongside PMT achieves better results with significant improvements in thrombus removal, approximately $62 \%$ lyses versus $26 \%$, therefore, there is reasonable census that CDT should be utilized alongside PMT unless there is a specific contra-indication to thrombolysis [37].

\section{Direct comparative studies PMT versus CDT}

Two retrospective cohort studies have reported comparative analysis PMT plus CDT in 150 patients (165 limbs). There were no significant differences in terms of thrombus clearance but PMT and CDT versus CDT alone resulted in shorter length of ICU in hospital stay, shorter treatment times, lower requirements for venogran (D) th all of the advantages of these have the potential to entail.

Owing to the shorter treatment time and less ICU and in-hospital stay, there is a huge reduction in cost, although it must be stated that the co extremely high (PMT plus CDT) - appiormately US $\$ 47,000 \pm \$ 19,000$ versus $\$ 85,000 \pm \$ 25,000$ per CDT patient [9]. 


\section{A practical approach to the management of a patient with acute DVT}

Just as catheter angiography in the assessment of peripheral vascular disease has largely been replaced by CT or MRI angiography so too has catheter venography been replaced by ultrasound and or CT or MR venography [38-41].

Ultrasound is widely used as the method of diagnosis of DVT; however, there is poor sensitivity and specificity for above groin DVT [42]. This is of critical importance in assessing anatomical variation of the IVC, large IVC volume loads and particularly for assessing the size of the existing iliac venous system, as commonly what is thought to be acute, is actually acute on chronic.

CT venography can provide clues, which will guide treatment and in the view of many institutions is essential.

The use of IVC filters is outside the scope of this article, it appears reasonable that they should be employed if there is a significant IVC thrombus load, if there is right ventricular dilatation, or any degree of cardiopulmonary strain [43,44].

An ultrasound is essential to assess the popliteal vein, as this is the vein chosen most commonly for access to the deep venous system of the lower extremity.

Owing to the uncertainty as to whether IVC filters are required, it is difficult to stratify patients into different categories. One such stratification is as follows, based on CT PA and CTV [45].

\section{Iliofemoral DVT with the following attributes}

- Group 1: no evidence of a pulmonary embolus (PE), IVC is clear, popliteal and calf veins are clear;

- Group 2: PE or IVC thrombus, popliteal and calf veins are clear;

- Group 3: no evidence of PE, IVC is clear, popliteal vein involved and calf vein thrombosis

- Group 4: PE or IVC thrombus, iliofemoral DVT extends down below the calf veins to involve the ankle.

In essence, each of the four groups will require slightly different treatment approaches. The aim is to aggressively treat the DVT by removing the thrombus in the shortest possible time using the safest possible technique whilst avoiding PE.

Those with PE or free-floating IVC thrombus will possibly require an IVC filter. Those with the popliteal vein clear can be treated by means of a pharmacomechanical thrombectomy device, while those with calf vein DVT will require CDT/EKOS.

Obviously local experience will dictate which particular method is chosen, this is partly dictated by the availability of close monitoring beds if CDT is to be employed, or if the institution's policy is that patients undergoing CDT do not require monitored beds, then this offers significant scope for reduction of cost.

\section{Conclusion}

PMT devices can be used as an adjunct to CDT for the aggressive treatment of acute DVT. Although there are no randomized control trials comparing mechanical thrombectomy versus CDT it is likely that the former may reduce complications by decreasing the dose of thrombolytic therapy, and also allows shorter treatment times with less use of monitored beds, low radiation dose, nephrotoxicity and cost.

Well-designed randomized control trials reported to consensus standard are required and the ATTRACT trial will provide this over the next few years.

\section{Future perspective}

The results of the ATTRACT trial will be pivotal in determining the importance of aggressive interventional therapy in DVT.

More aggressive therapy of DVT will likely become more common place.

It is most likely the PMT will increase in importance versus CDT.

More endovascular specialists will need to become expert in this space.

It is possible that treatment of acute DVT will assume the level of importance currently being aimed at stroke.

Financial \& competing interests disclosure

The author has no relevant affiliations or financial involvement with any organization or entity with a financial interest in or financial conflict with the subject matter or materials discussed in the manuscript. This includes employment, consultancies, honoraria, stock ownership or options, expert 
testimony, grants or patents received or pending, or royalties.

No writing assistance was utilized in the production of this manuscript.

\section{Bibliography}

1 Buller HR, Agnelli G, Hull RD et al. Antithrombotic therapy for venous thromboembolic disease: the 7th ACCP conference on antithrombotic and thrombolytic therapy. Chest 126(Suppl. 3), S401-S428 (2004).

2 Barritt DW, Jordan SC. Anticoagulant drugs in the treatment of pulmonary embolism a controlled trial. Lancet 1(7138), 1309-1312 (1960).

3 Grunwald MR, Hofmann LV. Comparison of urokinase, alteplase, and reteplase for catheter-directed thrombolysis of deep venous thrombosis. J. Vasc. Interv. Radiol. 15(4), 347-352 (2004).

4 Martinez Trabal JL, Comerota AJ, LaPorte $\mathrm{FB}$ et al. The quantitative benefit of isolated, segmental, pharmacomechanical thrombolysis (ISPMT) for iliofemoral venous thrombosis. J. Vasc. Surg. 48(6), 1532-1537 (2008).

5 Laiho MK, Oinonen A, Sugano N et al. Preservation of venous valve function after catheter-directed and systemic thrombolysis for deep venous thrombosis. Eur. J. Vasc. Endovasc. Surg. 28(4), 391-396 (2004).

6 AbuRahma AF, Perkins SE, Wulu JT et al. Iliofemoral deep vein thrombosis: conventional therapy versus lysis and percutaneous transluminal angioplasty and stenting. Ann. Surg. 233(6), 752-760 (2001)

7 Comerota AJ, Paolini D. Treatment of acute iliofemoral deep venous thrombosis: a strategy of thrombus removal. Eur. J. Vasc. Endovasc. Surg. 33(3), 351-362 (2007).

8 Kearon C. Natural history of venous thromboembolism. Circulation 107(23 Suppl. 1), S22-S30 (2003).

9 Snow V, Qaseem A, Barry P et al. Management of venous thromboembolism: a clinical practice guideline from the American College of physicians and the American Academy of family physicians. Ann. Fam. Med. 5(1), 74-80 (2007).

10 Winter M, Keeling D, Sharpen F et al. Haemostasis and thrombosis task force of the British Committee for standards in haematology. Procedures for the outpatient management of patients with deep venous thrombosis. Clin. Lab. Haematol. 27(1), 61-66 (2005).

11 Kearon C, Kahn SR, Agnelli G et al. Antithrombotic therapy for venous thromboembolic disease: American College of chest physicians evidence-based clinical practice guidelines (8th Edition). Chest 133(Suppl. 6), S454-S545 (2008).

12 Jaff MR, McMurtry MS, Archer SL et al. Management of massive and submassive pulmonary embolism, iliofemoral deep vein thrombosis, and chronic thromboembolic pulmonary hypertension: a scientific statement from the American heart association. Circulation 123(16), 1788-1830 (2011).

13 O'Donnell FF, Browse NL, Burnand KG et al. The socioeconomic effects of an iliofemoral venous thrombosis. J. Surg. Res. 22, 483-488 (1977).

14 Plate G, Eklöf B, Norgren L et al. Venous thrombectomy for iliofemoral vein thrombosis-10-year results of a prospective randomized study. Eur. J. Vasc. Endovasc. Surg. 5, 367-374 (1997).

15 Meissner MH, Manzo RA, Bergelin RO et al. Deep venous insufficiency: the relationship between lysis and subsequent reflux. J. Vasc. Surg. 18, 596-605 (1993).

16 Cochrane systematic review (2004)

17 Mewissen MW, Seabrook GR, Meissner MH et al. Catheter-directed thrombolysis for lower extremity deep venous thrombosis: report of a national multicenter registry. Radiology 211(1), 39-49 (1999).

18 Vedantham S, Millward SF, Cardella JF et al. Society of interventional radiology position statement: treatment of acute iliofemoral deep vein thrombosis with use of adjunctive catheter-directed intrathrombus thrombolysis. J. Vasc. Interv. Radiol. 20(Suppl. 7), S332S335 (2009).

19 Gandini R, Maspes F, Sodani G et al. Percutaneous ilio-caval thrombectomy with the Amplatz device: preliminary results. Eur. Radiol. 9(5), 951-958 (1999).

20 Delomez M, Beregi JP, Willoteaux S et al. Mechanical thrombectomy in patients with deep venous thrombosis. Cardiovasc. Intervent. Radiol. 24(1), 42-48 (2001).

21 Kasirajan K, Gray B, Ouriel K. Percutaneous AngioJet thrombectomy in the management of extensive deep venous thrombosis. J. Vasc. Interv. Radiol. 12(2), 179-185 (2001).

22 Vedantham S, Vesely TM, Parti N et al. Lower extremity venous thrombolysis with adjunctive mechanical thrombectomy. J. Vasc. Interv. Radiol. 13(10), 1001-1008 (2002).

23 Vedantham S, Vesely TM, Sicard GA et al. Pharmacomechanical thrombolysis and early stent placement for iliofemoral deep vein thrombosis. J. Vasc. Interv. Radiol. 15(6), 565-574 (2004).

24 Lin PH, Zhou W, Dardik A et al. Catheterdirect thrombolysis versus pharmacomechanical thrombectomy for treatment of symptomatic lower extremity deep venous thrombosis. Am. J. Surg. 192(6), 782-788 (2006).

25 Lee KH, Han H, Lee KJ et al. Mechanical thrombectomy of acute iliofemoral deep vein thrombosis with use of an arrow-trerotola percutaneous thrombectomy device. J. Vasc. Interv. Radiol. 17(3), 487-495 (2006).

26 Arko FR, Davis CM, Murphy EH et al. Aggressive percutaneous mechanical thrombectomy of deep venous thrombosis: early clinical results. Arch. Surg. 142(6), 513-518, discussion: 518-519 (2007).

27 Bush RL, Lin PH, Bates JT et al. Pharmacomechanical thrombectomy for treatment of symptomatic lower extremity deep venous thrombosis: safety and feasibility study. J. Vasc. Surg. 40 (5), 965-970 (2004).

28 Cynamon J, Stein EG, Dym RJ et al. A new method for aggressive management of deep vein thrombosis: retrospective study of the power pulse technique. J. Vasc. Interv. Radiol. 17(6), 1043-1049 (2006).

29 Gasparis AP, Labropoulos N, Tassiopoulos AK et al. Midterm follow-up after pharmacomechanical thrombolysis for lower extremity deep venous thrombosis. Vasc. Endovasc. Surg. 43(1), 61-68 (2009).

30 Jackson LS, Wang XJ, Dudrick SJ et al. Catheter-directed thrombolysis and/or thrombectomy with selective endovascular stenting as alternatives to systemic anticoagulation for treatment of acute deep vein thrombosis. Am. J. Surg. 190(6), 864-868 (2005).

31 Kim HS, Patra A, Paxton BE et al. Catheterdirected thrombolysis with percutaneous rheolytic thrombectomy versus thrombolysis alone in upper and lower extremity deep vein thrombosis. Cardiovasc. Intervent. Radiol. 29(6), 1003-1007 (2006).

32 Kim HS, Patra A, Paxton BE et al. Adjunctive percutaneous mechanical thrombectomy for lower-extremity deep vein thrombosis: clinical and economic outcomes. J. Vasc. Interv. Radiol. 17(7), 1099-1104 (2006).

33 Rao AS, Konig G, Leers SA et al. Pharmacomechanical thrombectomy for iliofemoral deep vein thrombosis: an alternative in patients with contraindications to thrombolysis. J. Vasc. Surg. 50(5), 1092-1098 (2009).

34 Shi HJ, Huang YH, Shen T et al. Percutaneous mechanical thrombectomy combined with catheter-directed thrombolysis in the treatment of symptomatic lower extremity deep venous thrombosis. Eur. J. Radiol. 71(2), 350-355 (2009).

35 Parikh S, Motarjeme A, McNamara T et al. Ultrasound-accelerated thrombolysis for the treatment of deep vein thrombosis: initial 
clinical experience. J. Vasc. Interv. Radiol. 19(4), 521-528 (2008).

36 Protack CD, Bakken AM, Patel N et al. Long-term outcomes of catheter directed thrombolysis for lower extremity deep venous thrombosis without prophylactic inferior vena cava filter placement. J. Vasc. Surg. 45(5), 992-997, discussion: 997 (2007).

37 Roditi G, Chandramohan S. Imaging of the deep venous system- venography/magnetic resonance venography/computed tomography venography. In: Vascular and Endovascular Challenges Update (Charing Cross). Greenhalgh RM (Ed.). BIBA Publishing, London, 432-447 (2010).

38 Orbell JH, Smith A, Burnand KG et al. Imaging of deep vein thrombosis. Br. J. Surg. 95(2), 137-146 (2008).

39 Kanne JP, Lalani TA. Role of computed tomography and magnetic resonance imaging for deep venous thrombosis and pulmonary embolism. Circulation 109(12 Suppl. 1), S15-S121 (2004).

40 Katz DS, Loud PA, Bruce D et al. Combined CT venography and pulmonary angiography: a comprehensive review. Radiographics 22(Spec No), S3-S24 (2002).

41 Messina LM, Sarpa MS, Smith MA et al. Clinical significance of routine imaging of iliac and calf veins by color flow duplex scanning in patients suspected of having acute lower extremity deep venous thrombosis. Surgery 114(5), 921-927 (1993).

42 Lohan DG, Cronin CG, Meehan CP. Massive pulmonary embolus with hemodynamic compromise: therapeutic options. Emerg. Radiol. 13(4), 161-169 (2007).

43 O'Sullivan GJ. Isolated pharmacomechanical thrombolysis using the trellis system. In: Vascular and Endovascular Challenges Update (Charing Cross). Greenhalgh RM (Ed.). BIBA Publishing, London, 552-564 (2010).

44 Kasirajan K, Gray B, Ouriel K. Percutaneous AngioJet thrombectomy in the management of extensive deep venous thrombosis. J. Vasc. Interv. Radiol. 12(2), 179-185 (2001).

45 Vedantham S, Thorpe PE, Cardella JF et al. Quality improvement guidelines for the treatment of lower extremity deep vein thrombosis with use of endovascular thrombus removal. J. Vasc. Interv. Radiol. 20 (Suppl. 7), S227-S239 (2009).

46 O'Sullivan GJ, Lohan DG, Gough N et al. Pharmacomechanical thrombectomy of acute deep vein thrombosis with the Trellis-8 isolated thrombolysis catheter. J. Vasc. Interv. Radiol. 18(6), 715-724 (2007).

47 Vedantham S, Vesely TM, Parti N et al. Lower extremity venous thrombolysis with adjunctive mechanical thrombectomy. J. Vasc. Interv. Radiol. 13(10), 1001-1008 (2002).

48 O'Sullivan GJ. The role of interventional radiology in the management of deep venous thrombosis: advanced therapy. Cardiovasc. Intervent. Radiol. 34(3), 445-461 (2011).

49 Kanne JP, Lalani TA. Role of computed tomography and magnetic resonance imaging for deep venous thrombosis and pulmonary embolism. Circulation 109(12 Suppl. 1), S15-S121 (2004).
50 Katz DS, Loud PA, Bruce D et al. Combined CT venography and pulmonary angiography: a comprehensive review. Radiographics 22(Spec No), S3-S24 (2002).

\section{Website}

101 The ATTRACT study. www.attract.wustl.edu

102 NCTS 00970619 DUTCH CAVA-trial: Catheter versus anticoagulation alone for acute primary (Ilio) Femoral DVT. (NL28394). www.clinicaltrials.gov/ct2/show/ NCT00970619

103 NCTS 00640731 Safety and efficacy study of omniwave endovascular system to treat deep vein thrombosis (SONIC I). www.clinicaltrials.gov/ct2/show/ NCT00640731

104 NCTS 00778336 . A prospective observational registry of peripheral use of AngioJet rheolytic thrombectomy with mid-length catheters. www.clinicaltrials.gov/ct2/show/ NCT00778336

105 NCTS 00790335 Acute venous thrombosis: thrombus removal with adjunctive catheterdirected thrombolysis (ATTRACT). www.clinicaltrials.gov/ct2/show/ NCT00790335 